\title{
Laparoscopic repair of incisional and ventral hernias with the new type of meshes: randomized control trial
}

\author{
Vladimir V. Grubnik, Aleksandra V. Grubnik, Kseniya O. Vorotyntseva \\ Department of General Surgery, Odessa National Medical University, Odessa Regional Hospital, Odessa, Ukraine \\ Videosurgery Miniinv 2014; 9 (2): 145-151
}

DOI: $10.5114 /$ wiitm.2014.41623

\begin{abstract}
Introduction: Laparoscopic incisional and ventral hernia repair (LIVHR) was first reported by Le Blanc and Booth in 1993. Many studies are available in the literature that have shown that laparoscopic repair of incisional and ventral hernia is preferred over open repair because of lower recurrence rates (less than 10\%), less wound morbidity, less pain, and early return to work.

Aim: To identify the long-term outcomes between the different types of meshes and two techniques of mesh fixation, i.e., tacks (method Double crown) and transfascial polypropylene sutures.

Material and methods: A total of 92 patients underwent LIVHR at our department between January 2009 and August 2012. The hernias were umbilical in 26 patients, paraumbilical in 15 patients and incisional in 51 patients. All patients admitted for LIVHR were randomized to either group I (tacker fixation of ePTFE meshes) or group II (suture fixation of meshes with nitinol frame) using computer-generated random numbers with block randomization and sealed envelopes for concealed allocation.

Results: The mean mesh fixation time was significantly higher in the tacker fixation group (117 $\pm 15 \mathrm{~min} v \mathrm{vs}$. $72 \pm 6 \mathrm{~min}$, $p<0.01)$. There were no conversions in either group. The median postoperative hospital stay was $3.5 \pm 1.5$ days. All patients were followed up at 1, 3, 6, 12 and every 6 months thereafter postoperatively. There were 5 recurrences in the study population. In group I there were 4 patients with recurrence, and only 1 patient in the group with meshes with a nitinol frame.

Conclusions: Meshes of the new generation with a nitinol framework can significantly improve laparoscopic ventral hernia repair. The fixation of these meshes is very simple using 3-4 transfascial sutures. The absence of shrinkage of these meshes makes the probability of recurrence minimal. Absence of tackers allows postoperative pain to be minimized. We consider that these new meshes can significantly improve laparoscopic ventral hernia repair.
\end{abstract}

Key words: laparoscopic hernia repair, meshes, ventral hernia.

\section{Introduction}

Laparoscopic incisional and ventral hernia repair (LIVHR) was first reported by Le Blanc and Booth in 1993 [1]. Many studies are available in the literature that have shown that laparoscopic repair of incisional and ventral hernia is preferred over open repair because of lower recurrence rates (less than 10\%), lower wound morbidity, less pain, and early return to work
$[2,3]$. The technique of laparoscopic repair of incisional and ventral hernia has almost been standardized, and the issues, such as access to the abdominal cavity, mesh size, and extent of overlap, have been resolved. However, there are still areas of debate; they include the ideal prosthetic material to be used, management of hernia defect, and the technique of fixation of the mesh to the abdominal wall [4-6].

\section{Address for correspondence:}

Department of General Surgery, Odessa National Medical University, Odessa Regional Hospital, 26 Zabolotnogo St, Odessa, Ukraine, phone: +380487500116, e-mail: solnce-sun@ukr.net 
The laparoscopic technique of ventral hernia repair requires placement of the prosthesis in the peritoneal cavity, where there is a greater risk of adhesion formation, erosion of the mesh into the intestine, and fistula formation [3, 7-9].

The characteristics of an ideal mesh should include adhesion prevention on one hand and excellent fibrous ingrowth on the other hand. To achieve this, manufacturers have constructed mesh prostheses composed of layers. Each layer is designed to meet the ideal characteristics of either the parietal or the visceral surface of the mesh $[1,10,11]$.

Various prostheses are available for the repair of abdominal wall defects. Each type of mesh has unique properties that affect the development of adhesions and tissue ingrowth. Polypropylene mesh (PPM) (Prolene, Ethicon Endosurgery, Cincinnati, OH, USA), for example, is one of the original hernia prostheses and has properties that lead to excellent fibrous ingrowth. Expanded polytetrafluoroethylene (ePTFE) causes less reaction, and many surgeons advocate its use because of a perception that it is less adhesiogenic $[10,12,13]$.

Composite meshes are designed with a different material for each of two layers - one layer to minimize or prevent adhesions to the visceral surface and one layer to maximize fibrous ingrowth on the parietal surface. Parietex Composite (PCO) (Sofradim International, Trevoux, France) is a three-dimensional multifilament polyester mesh bonded to a continuous, smooth hydrophilic and absorbable collagen film that overlaps the cut edge of polyester by $5 \mathrm{~mm}$. This mesh was designed to preserve brisk parietal tissue ingrowth while preventing adhesions to the visceral surface of the mesh [14-16].

A new generation of meshes has been created. The self-expanding MMDI mesh is constructed by

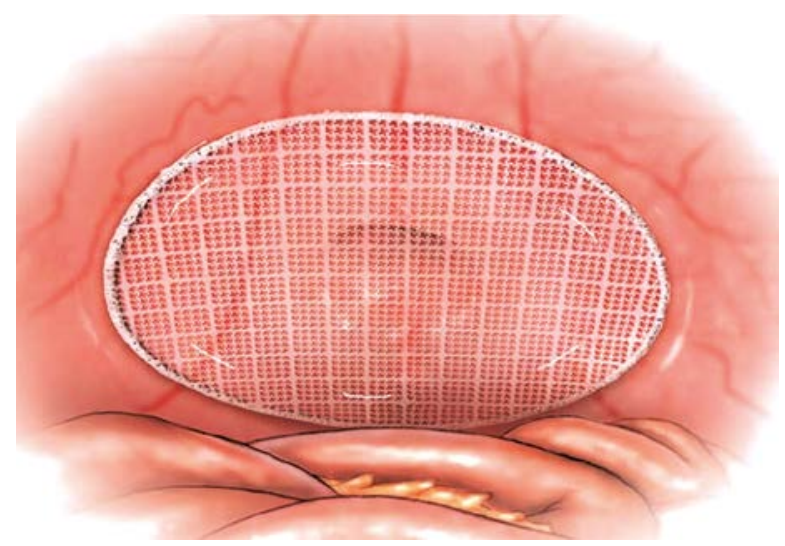

Photo 1. MMDI mesh with nitinol frame combining a super-elastic nitinol frame with an advanced ePTFE mesh (Photo 1). It is thin, macroporous, transparent, condensed polytetrafluoroethylene (ePTFE) implant material that combines the favorable tissue ingrowth and healed strength characteristics of large pore monofilament polypropylene surgical mesh with the biocompatibility attributes of expanded PTFE. The nitinol frame keeps the mesh flat and firmly positioned against the abdominal wall while reducing the incidence of mesh shrinkage [10, 17-19].

Due to the variety of prosthetic mesh materials now available, the general surgeon faces the difficult decision of determining which material is the most appropriate for a particular hernia repair application.

There is no description of a standard technique of mesh fixation. Common methods of mesh fixation are metallic tacks with or without transfascial sutures and transfascial sutures alone [18, 20].

Various studies in the literature have used mesh fixation with only tacks or only sutures, and with tacks and sutures together [18, 21]. Although mesh fixation with tacks is convenient and time saving, the tensile strength of a mesh fixed by transfascial sutures has been shown to be up to 2.5 times greater than when fixed by tacks. Transfascial sutures penetrate all layers of the abdominal wall, thereby enabling fixation of the mesh to the entire fasciomuscular layer of the abdominal wall. The number of sutures, the distance between the sutures, the sites on the mesh where they are placed, and whether nonabsorbable or absorbable suture material is used are all technical issues under debate.

It is necessary to remember that the laparoscopic approach is a valuable option in the management of incisional hernias, but it requires experience in laparoscopic surgery due to its specific learning curve [19]. The recurrence rate is higher when surgeons are less experienced and technically less proficient. In addition, the risk of enterotomy, with potentially disastrous outcomes, is probably related to the learning curve $[16,22]$.

\section{Aim}

The aim of the study was to identify the longterm outcomes between the different type of meshes and two techniques of mesh fixation. i.e., tacks (double crown method) and transfascial polypropylene sutures. 


\section{Material and methods}

A total of 92 patients underwent LIVHR at our department between January 2009 and August 2012. There were 39 men and 53 women of a mean age of 56 years (range: $22-80$ years). The mean area of defect was $68.46 \mathrm{~cm}^{2}$ (range: $8-197 \mathrm{~cm}^{2}$ ). The mean body mass index (BMI) of patients of the two groups was $28.4 \mathrm{~kg} / \mathrm{m}^{2}$ (range: 18-39). The hernias were umbilical in 26 patients, paraumbilical in 15 patients and incisional in 51 patients.

Patients meeting the inclusion criteria - older than 18 years, presenting with ventral hernia (incisional) with hernia diameter of $12 \mathrm{~cm}$ or less at its greatest diameter, and fit for surgery - were consecutively enrolled in the study. Operations were performed in our clinic after written consent had been obtained. Exclusion criteria were patients with previous mesh implantation, significant comorbidity, $\mathrm{BMI}>45 \mathrm{~kg} / \mathrm{m}^{2}$, pregnancy, contaminated abdominal cavity or long-term use of immunosuppressive agents.

All patients received a complete physical examination and standard laboratory and radiological work-up prior to surgery. Anesthesiological risk was classified according to the American Society of Anesthesiologists (ASA) classification. In our study 11 patients were with ASA I, 62 with ASA II and 19 with ASA III.

All patients admitted for LIVHR were randomized to either group I (tacker fixation of PTFEe meshes) or group II (suture fixation of meshes with nitinol frame) using computer-generated random numbers with block randomization and sealed envelopes for concealed allocation.

General anesthesia was used with endotracheal intubation in all patients. After creation of pneumoperitoneum with a Veress needle at the umbilicus or left hypochondrium (Palmer's point), a 10-mm laparoscopic port for a telescope was introduced in the left flank at the level of the umbilicus. Two additional 5-mm ports were placed as deemed appropriate depending upon the location of the hernia defect. Omental and bowel adhesions were taken down using monopolar diathermy, harmonic scalpel or scissors. The hernia sac was left intact. In neither case was suturing of the hernia defect performed, to prevent tension of the tissue.

In group I PTFEe meshes (Gore-Tex) (33 patients) of appropriate size were used to cover the defect with an overlap of at least $4-5 \mathrm{~cm}$ on all sides. In group I, the mesh was fixed with $5-\mathrm{mm}$ metal tackers (Ethicon). Double-crown technique was applied for placement of tacks, i.e., two circular rows of tacks, with the first row at the extreme periphery of the mesh all around (single crown), and an inner row of tacks to reinforce the middle portion of the mesh closer to the margin of the defect (double crown) [21].

In group II meshes with MMDI nitinol frame (41 patients) were used. Due to the super-elastic nitinol frame the device was folded and inserted laparoscopically through an access port to the abdominal cavity. In the abdominal cavity the nitinol frame expanded the mesh back to its original shape. Mesh placement was facilitated by placing a centering suture through the center of the defect, then as usual four (in 3 patients 3 sutures and in 4 patients 5 sutures) corner 1-0 polypropylene transfascial sutures were tied subcutaneously through the abdominal wall.

The demographic profile, preoperative hernia characteristics, intraoperative variables, and complications were noted. Pain score on the visual analogue scale (VAS) was evaluated at $1 \mathrm{~h}, 6 \mathrm{~h}$, and $24 \mathrm{~h}$ after surgery. After discharge the patients were followed up in the outpatient clinic at 1 week, 1 month, 3 months, 6 months and 12 months. Late postoperative complications such as seroma, wound infection or any other problems were noted.

\section{Statistical analysis}

Data were collected and managed using Microsoft Excel (Microsoft, Seattle, WA, annexure 8). Unpaired Student $t$-test was used to determine the significance of difference between two independent groups among continuous variables, such as age, defect size, and operative time. For skewed data, a corresponding nonparametric test, Mann-Whitney test, was applied to evaluate the difference between the two independent groups. For qualitative data, $\chi^{2}$ test was used to determine a significant difference in proportion between the two groups. Value of $p<0.05$ was considered significant. All statistical analyses were performed using the statistical package SPSS 14.

\section{Results}

A total of 92 patients with incisional and primary ventral hernia were preoperatively randomized 
Table I. Demographic data and hernia characteristics

\begin{tabular}{|lcccc|}
\hline Parameter & $\begin{array}{c}\text { Study group } \\
(n=92)\end{array}$ & $\begin{array}{c}\text { Group I } \\
(n=43)\end{array}$ & $\begin{array}{c}\text { Group II } \\
(n=49)\end{array}$ & Value of $p$ \\
\hline Age, mean \pm SD [years] & $52.1 \pm 13.6$ & $51.8 \pm 13.2$ & $52.4 \pm 12.8$ & 0.98 \\
\hline \begin{tabular}{l} 
Gender: \\
\hline Male
\end{tabular} & 31 & 15 & 16 & 0.12 \\
\hline Female & 61 & 29 & 33 & 0.34 \\
\hline BMI, mean \pm SD $\left[\mathrm{kg} / \mathrm{m}^{2}\right]$ & $31.7 \pm 7.7$ & $32.8 \pm 5.6$ & $31.9 \pm 7.5$ & 15 \\
\hline \begin{tabular}{l} 
Type of hernia: \\
\hline Umbilical
\end{tabular} & 26 & & & 8 \\
\hline $\begin{array}{l}\text { Paraumbilical } \\
\text { Incisional }\end{array}$ & 15 & 71 & 28 & \\
\hline
\end{tabular}

Table II. Intraoperative and postoperative data

\begin{tabular}{|c|c|c|c|c|}
\hline Parameter & $\begin{array}{l}\text { Study group } \\
\quad(n=92)\end{array}$ & $\begin{array}{l}\text { Group I } \\
(n=43)\end{array}$ & $\begin{array}{l}\text { Group II } \\
(n=49)\end{array}$ & Value of $p$ \\
\hline Operative time, mean \pm SD [min] & $98.6 \pm 7.4$ & $117 \pm 15$ & $72 \pm 6$ & $<0.01$ \\
\hline Follow-up, mean \pm SD (range) [months] & $13.7 \pm 7.0$ & $14.8 \pm 5.9$ & $12.9 \pm 3.6$ & 0.34 \\
\hline Postoperative hospital stay [days] & $3.5 \pm 1.5$ & $4.5 \pm 2$ & $2.5 \pm 1.5$ & $<0.01$ \\
\hline Seroma & 8 & 7 & 1 & $<0.01$ \\
\hline Hematoma & 8 & 8 & 0 & $<0.01$ \\
\hline Recurrences & 5 & 4 & 1 & $<0.01$ \\
\hline
\end{tabular}

to the two groups. Forty-three patients were randomized to group I (tacker fixation of meshes Gore-Tex) and 49 were randomized to group II (suture fixation of MMDI meshes). The two groups were well matched in terms of age, sex, and hernia characteristics (Table I).

The mean body mass index (BMI) was $31.7 \pm 7.7$ $\mathrm{kg} / \mathrm{m}^{2}$. The distribution of defect sites was compared in the two groups (Table I). The mean defect size was $121.7 \pm 79.7 \mathrm{~cm}^{2}$. The mean operative time and mesh fixation time were statistically and significantly higher in group I (Table II). Mesh fixation time was calculated from the time of introduction of mesh into the abdomen until release of the final tack/tying of the last suture and the operative time was calculated from skin incision for Veress insertion until firing of the last skin suture for skin closure. The mean mesh fixation time was significantly higher in the tacker fixation group $(117 \pm 15 \mathrm{~min}$ vs. $72 \pm 6 \min , p<0.01)$. There were no conversions in either group. The median postoperative hospital stay was $3.5 \pm 1.5$ days. There was a statistically significant difference in hospital stay between the two groups ( $4.5 \pm 2$ days vs. $2.5 \pm 1.5$ days; $p<0.01)$. All patients were followed up at 1, 3, 6, 12 and every 6 months thereafter postoperatively. The mean follow-up period was $26 \pm 9$ months.

In group I, seromas developed in 7 patients and in group II only in 1 patient $(p<0.05)$. In the group with tacker fixation there were 8 patients with hematomas $(p<0.01)$. The main question of the study was recurrence rate. There were 5 recurrences in the study population; in group I there were 4 patients with recurrence, and only 1 patient in the group with meshes with a nitinol frame (Table II).

Postoperative pain was assessed using a visual analogue scale (0-10). The pain scores were adjusted for gender and analyzed, because gender distri- 
bution was statistically different in the two groups. The tacker fixation group had significantly higher pain scores at $1 \mathrm{~h}, 6 \mathrm{~h}$, and 1 month after operation. At 3 months postoperatively, 17 patients still complained of mild pain at the operation site. Twelve patients in the tacker group I and 5 patients in the suture group II had pain. The pain was intermittent, associated with activity involving abdominal musculature, usually with a sudden change in posture.

In our study we did not find any difference between the two groups of patients. It can be explained by the use of miniinvasive laparoscopic techniques in both groups.

Different fixation techniques are associated with varying degrees of early postoperative and chronic pain, which may affect the quality of life (QOL) postoperatively. In our study, there was a significant improvement in QOL from the preoperative to postoperative period in both groups, but the two groups showed no significant difference in any of the domains postoperatively. Thus, the QOL improvement is independent of technique of fixation, whether tacks with corner sutures or sutures alone.

\section{Discussion}

One of the main questions is adhesion formation of the mesh. Numerous publications, including those of an experimental nature, concentrate mainly on the extent and pathology of adhesions. In this regard, Gore-Tex PTFE meshes prove to be considerably inferior to lightweight meshes with a nitinol frame.

Greater attention must be paid to the effect of shrinkage. The pathophysiologic reactions involved in this phenomenon are extremely complex. Shrinkage of the material is actually the last link in the body and is the chain of reactions to the foreign material. These reactions appear to be clearly related to the site of mesh placement, and also to the amount and structure of the material [10]. This would explain the observation that over the long term, Gore-Tex PTFE meshes fixed in an identical manner show considerably less tendency to shrink than lightweight MMDI meshes, due to the nitinol frame.

The ideal design of a mesh that prevents adhesion formation and promotes incorporation and tensile strength probably complies with the basic rules. The visceral side of the mesh should be smooth, non-erosive, anti-adhesive, and not easily susceptible to infection. This visceral barrier should be pres- ent for at least 1 week because within this time adhesion formation of the frame takes place [7]. The ventral side of the mesh should be macroporous, allowing for fibroblast ingrowth, and a foreign body reaction actually may be necessary for incorporation and high tensile strength. Continued severe inflammation, on the other hand, actually may decrease mesh incorporation and tensile strength [13].

The various methods of mesh fixation described in the literature are staples [1], tacks in a single crown [23] and transfascial sutures either alone [18] or in combination with tacks [19]. Currently, the most popular method of mesh fixation used worldwide is the use of tacks. Mesh fixation with only transfascial sutures has not been used by many authors. The only large study where transfascial suture fixation has been applied was done by Chelala et al. [18]. They obtained excellent results with a recurrence rate of $1.5 \%$ at a mean follow-up of 28 months. Not many studies in the literature compare various methods of mesh fixation in laparoscopic incisional and ventral hernia repair.

LeBlanc et al. [1, 20, 22] recommended that the minimum mesh overlap should be $4-5 \mathrm{~cm}$ if transfascial sutures were not used and at least $3 \mathrm{~cm}$ when transfascial sutures were used, and also suggested that the transfascial sutures should not be placed more than $5 \mathrm{~cm}$ apart. We used a minimum mesh overlap of $4-5 \mathrm{~cm}$ on all sides, and tacks or transfascial sutures were placed at a distance of 2$3 \mathrm{~cm}$ from each other for mesh fixation.

The use of only transfascial suture fixation has not become very popular because of certain drawbacks. Suture fixation is time consuming. It is difficult to insert these transfascial sutures in certain anatomical areas such as the pelvis, above the subcostal margin, and laterally in the flanks.

We did not perform suturing of the hernia defect before placement of the mesh. The "tension free" technique allows one to avoid excessive tension of tissue and prevent recurrences in the postoperative period [18].

In group II as usual we used four transfascial sutures for fixation of the mesh with a nitinol frame to the abdominal wall, in 3 patients (group II) we used only 3 sutures, and in four patients we had to use 5 sutures for fixation to avoid bowel obstruction. In our study thanks to the individual approach we could prevent dangerous complications that can lead to bowel obstruction. 
Postoperative pain after LIVHR seems to be a relevant complaint during the early postoperative period, leading to increased consumption of pain medication, delayed bowel function, and extended hospital stay [17]. The incidence of chronic pain after laparoscopic incisional hernia repair has been reported to be approximately $1-3 \%$ in the literature [7]. The pain is largely related to mesh fixation with tacks or sutures. The pain due to fixation is different from that at the port sites. The postoperative pain produced by the fixation techniques could play an important role in deciding between sutures and tacks for mesh fixation. In one study, with tacker fixation only, persistent postoperative pain was reported to occur in $7.4 \%$ of the patients [7].

Some studies have suggested that transfascial suture fixation causes more pain [15]. Sutures penetrate through the full thickness of the abdominal wall musculature and fascia. This has been theorized to cause local muscle ischemia resulting in severe pain postoperatively [24]. Beldi et al. [4] did a randomized, controlled study comparing single crown tacker without corner suture fixation with only suture fixation and found more pain in the suture fixation group within the first 6 weeks of the postoperative period while there was no difference in pain at 6 months.

In our study, the pain scores in the suture fixation group were significantly lower at $1 \mathrm{~h}, 6 \mathrm{~h}$, and 1 month postoperatively ( $p<0.001$, Table II).

This could be explained by the fact that the patients were taking pain killers at $24 \mathrm{~h}$ and at 1 week, whereas at 1 month most patients stopped taking pain killers, thus making the difference in pain apparent again at 1 month. There was no statistically significant difference in the pain scores at and after 3 months postoperatively. The patients in the suture fixation group in our study had significantly lower pain scores because of the loose tying of the sutures after partial deflation, as suggested by Chelala et al. [18].

The pain is usually intermittent, associated with activity involving abdominal musculature, and occurs with a sudden change in posture. This could be explained by fibrosis entrapping the nerve or neuroma formation at the specific site of tack or suture fixation, which is stretched/compressed during specific muscle movement, causing long-term pain in these patients.

Hope et al. [8] reported laparoscopic repair to be better than open repair. Some other studies have shown an improvement in QOL after laparoscopic repair [8].

Different fixation techniques are associated with varying degrees of early postoperative and chronic pain, which may affect the quality of life postoperatively. In our study, there was a significant improvement in QOL from the preoperative to postoperative period in both groups, but the two groups showed no significant difference in any of the domains postoperatively. Thus, the QOL improvement is independent of technique of fixation, whether tacks with corner sutures or sutures alone.

Return to daily activities and to work is an important measure for assessment of success of any surgical intervention. However, laparoscopic incisional and ventral hernia is different from other laparoscopic procedures, in terms of considerably early postoperative pain, which may prolong the return to normal activities and return to work in many patients. In our study, we found that patients with suture fixation returned earlier to activity especially with a significant difference in resumption of usual activities $(p<0.001)$ compared with the tacker group. The increase of hospital stay in group I was associated with the development of pain expressed by the use of many tackers and the development of seroma. We found that in group II patients pain was significantly less, due to the small number of sutures.

There was no significant difference in time taken to start walking, moving about freely, dressing oneself, driving a vehicle/doing kitchen work, lifting objects, intercourse, resuming work, working unrestricted, or time taken off work. The mean time to resume work was 12 days, which is consistent with previous reports [12].

The recurrence rate in our study was low; four patients had recurrence with an average follow-up of 42 months. All patients were in the tacker group. These patients are females older than 50 years, BMI $>31 \mathrm{~kg} / \mathrm{m}^{2}$ with lower abdomen midline incisional defect following gynecologic surgery. The most probable cause of recurrence is mesh shrinkage or intrinsic factors related to the patient, such as abdominal wall weakness. The recurrences occurred on the lateral side of the mesh.

Beldi et al. [4] reported that mesh shrinkage was greater in the tack group compared with the suture group. Variable recurrence rates were reported in the literature with the use of different mesh fixa- 
tion techniques. In a collective review of 23 studies and 12 comparative studies by LeBlanc et al. [1, 20], mesh fixation with sutures only resulted in the lowest recurrence rate $(0.8 \%)$ compared with that by tacks alone (1.5\%). Mesh fixation with tacks and sutures resulted in a recurrence rate of $3.5 \%$ [21]. Carbajo et al. [21] and Gillian et al. [14] used only a double crown of tacks for mesh fixation. Carbajo et al. [21] added a recurrence rate of $4.4 \%$ during a follow-up period of 44 months and Gillian et al. [14] had a recurrence rate of $1 \%$ during a mean follow-up of 27 months.

\section{Conclusions}

The suture fixation method is a cost-effective alternative to tacker fixation in patients with small- to medium-sized defects in laparoscopic incisional and ventral hernia repair. Meshes of the new generation with a nitinol framework can significantly improve laparoscopic ventral hernia repair. The fixation of these meshes is very simple using 3-4 transfascial sutures. The absence of shrinkage of these meshes makes the probability of recurrence minimal. Absence of tackers allows postoperative pain to be avoided. We consider that these new meshes can significantly improve laparoscopic ventral hernia repair.

\section{References}

1. LeBlanc KA, Booth WV. Laparoscopic repair of incisional hernias using ePTFE: preliminary findings. Surg Laparosc Endosc 1993; 3: 39-41.

2. Barbaros U, Asoglu O, Seven R, et al. The comparison of laparoscopic and open ventral hernia repairs: a prospective randomized study. Hernia 2007; 11: 51-6.

3. Olmi S, Scaini A, Erba L, Croce E. Use of fibrin glue (Tissucol) in laparoscopic repair of abdominal wall defects: preliminary experience. Surg Endosc 2007; 21: 409-13.

4. Beldi G, Wagner M, Bruegger LE, et al. Mesh shrinkage and pain in laparoscopic ventral hernia repair: a randomized clinical trial comparing suture versus tack mesh fixation. Surg Endosc 2011; 25: 749-55.

5. Dąbrowiecki S, Pierściński S, Wojciech S. The Glubran 2 glue for mesh fixation in Lichtenstein's hernia repair: a double-blind randomized study. Videosurgery Miniinv 2012; 7: 96-104.

6. Pielaciński K, Szczepanik AB, Misiak A, Wróblewski T. Randomized clinical trial comparing inguinal hernia repair with Lichtenstein technique using non-absorbable or partially absorbable mesh. Preliminary report. Videosurgery Miniinv 2011; 6: 190-206.

7. Jenkins ED, Yom V, Melman L, et al. Prospective evaluation of adhesion characteristics to intraperitoneal mesh and adhesiolysis-related complications during laparoscopic re-exploration after prior ventral hernia repair. Surg Endosc 2010; 24: 3002-7.
8. Hope WW, Lincourt AE, Newcomb L. Comparing quality of life outcomes in symptomatic patients undergoing laparoscopic or open ventral hernia repair. J Laparosc Adv Surg Tech 2008; 1: 567-71.

9. Pesta W, Kurpiewski W, Łuba M, et al. Single incision laparoscopic surgery transabdominal pre-peritoneal hernia repair case report. Videosurgery Miniinv 2012; 7: 137-9.

10. Torres-Villalobos G, Sorcic L, Ruth GR, et al. Evaluation of the rebound hernia repair device for laparoscopic hernia repair. JSLS 2010; 14: 95-102.

11. Jamry A, Jałyński M, Śmigielski J, Brocki M. Shrinkage of dynamesh IPOM mesh in 6-week follow-up - an experimental study. Videosurgery Miniinv 2011; 6: 19-23.

12. Erikssen JR, Poornoroozy P, Jorgensen LN, et al. Pain, quality of life and recovery after laparoscopic ventral hernia repair. Hernia 2007; 13: 13-21.

13. Matthews BD, Mostafa G, Carbonell AM, et al. Evaluation of adhesion formation and host tissue response to intra-abdominal polytetrafluoroethylene mesh and composite prosthetic mesh. J Surg Res 2005; 123: 227-34.

14. Gillian GK, Geis WP, Grover G. Laparoscopic incisional and ventral hernia repair (LIVH): an evolving outpatient technique. JSLS 2002; 6: 315-22.

15. Perrone JM, Soper NJ, Eagon LC. Perioperative outcomes and complications of laparoscopic ventral hernia repairs. Surgery 2005; 138: 715-6.

16. Sroka G, Feldman LS, Vassiliou M, et al. Fundamentals of laparoscopic surgery simulator training to proficiency improves laparoscopic performance in the operating room: a randomized controlled trial. Am J Surg 2010; 199: 115-20.

17. Bellows C, Berger D. Infiltration of suture sites with local anesthesia for management of pain following laparoscopic ventral hernia repairs: a prospective randomized trial. JSLS 2006; 10: 345-50.

18. Chelala E, Thoma M, Tatete B, et al. The suturing concept for laparoscopic mesh fixation in ventral and incisional hernia repair: mid-term analysis of 400 cases. Surg Endosc 2007; 21: 391-5.

19. Heniford BT, Park A, Ramshaw BJ, Voeller G. Laparoscopic repair of ventral hernias: nine years' experience with 850 consecutive hernias. Ann Surg 2003; 238: 391-9.

20. LeBlanc KA. Laparoscopic incisional hernia repair: are trans-fascial sutures necessary? A review of the literature. Surg Endosc 2007; 21: 508-13.

21. Carbajo MA, Martin del Olmo JC, Blanco JI, et al. Laparoscopic approach to incisional hernia. Surg Endosc 2003; 17: 118-22.

22. Leblanc KA, Elieson MJ, Corder JM III. Enterotomy and mortality rates of laparoscopic incisional and ventral hernia repair: a review of the literature. JSLS 2007; 11: 408-14.

23. Kirshtein B, Lantsberg L, Avinoach E, et al. Laparoscopic repair of large incisional hernias. Surg Endosc 2002; 16: 1717-9.

24. Nguyen SQ, Divino CM, Buch KE, et al. Postoperative pain after laparoscopic ventral hernia repair: a prospective comparison of sutures versus tacks. JSLS 2008; 12: 113-6.

Received: 14.04.2012, accepted: 5.08.2013. 